\title{
SPALLATION NEUTRON SOURCE BEAM CURRENT MONITOR ELECTRONICS ${ }^{1}$
}

\author{
Martin Kesselman, BNL, Upton, NY 11973, USA
}

\begin{abstract}
The Spallation Neutron Source (SNS) to be constructed at ORNL is a collaboration of six laboratories. Beam current monitors for SNS will be used to monitor Hminus and $\mathrm{H}$-plus beams ranging from the $15 \mathrm{~mA}$ (tuneup in the Front End and Linac) to over 60 A fully accumulated in the Ring. The time structure of the beams to be measured range from $645 \mathrm{nsec}$ "mini" bunches, at the $1.05 \mathrm{MHz}$ ring revolution rate, to an overall $1 \mathrm{mS}$ long macro pulse. Beam current monitors (BCMs) for SNS have requircments depending upon their location within the system. The development of a general approach to satisfy requirements of various locations with common components is a major design objective. This paper will describe the development of the beam current monitors and electronics.
\end{abstract}

\section{BACKGROUND}

This is the third in a series of papers [1,2] describing the progress of the beam current monitoring system. In summary of the references [1 \& 2]: the SNS consists of an $\mathrm{H}$ Ion source, RFQ and MEBT (the Front End) provided by LBNL, the Linac consisting of a warm section and a super-conducting section developing $1 \mathrm{Gev} \mathrm{H}$, provided by LANL and TJNAL, the HEBT (high energy beam transport), Accumulator Ring and RTBT (Ring to beam target) provided by BNL, and the target provided by ORNL.

The MEBT, Linac, and HEBT design current is typically a $57 \mathrm{~mA}$ peak pulse of about $1 \mathrm{~ms}$ duration (macro-pulse) chopped at the Ring revolution frequency near $1.05 \mathrm{MHz}$, with a repetition rate of $60 \mathrm{~Hz}$. This pulse, however, could be reduced to near $15 \mathrm{~mA}$ during studies. The chopped "mini-pulses" will consist of a $645 \mathrm{~ns}$ long bunch and a $300 \mathrm{~ns}$ gap. The "micro-pulse" structure of $402.5 \mathrm{MHz}$, which exists in the Front End, Linac, and HEBT, and shortly after injection in the Ring will not be observed with the beam current monitors (BCMs).

The Ring will accumulate the mini-pulses over approximately $1 \mathrm{~ms}$, about 1000 turns, resulting in a growing beam current that will reach near 57A peak. The entire beam is dumped into the RTBT yielding a single pulse of about $645 \mathrm{~ns}$. The RTBT carries this beam to the target to produce neutrons. The electronics in the Ring and RTBT must operate over a large dynamic range.

\footnotetext{
${ }^{1}$ Work performed under the auspices of the U.S. Department of Energy
}

\section{BCM SYSTEM DESCRIPTION}

The basic pick-up element is a Bergoz ${ }^{\circledR}$ FCT (fast current transformer [3]. This transformer has been chosen with 50 turns, providing a trade-off between sensitivity, rise-time and droop. A droop of $0.1 \% / \mu \mathrm{s}$, rise time of $<1 \mathrm{~ns}$, and sensitivity of $0.5 \mathrm{~V} / \mathrm{A}$ is the target goal. A technique to restore the droop to $0.1 \% / \mathrm{ms}$ will be discussed later. The device has been built with an additional 10 turn winding to provide a calibration/test input capability. A first article has been received and tested. It provided a high frequency cut-off at about $800 \mathrm{MHz}$, consistent with a rise time of about $440 \mathrm{ps}$. The sensitivity measured 0.47V/A (indicates a $0.5 \mathrm{~dB}$ loss due to the internal matching resistor configuration used in it's manufacture). Droop was measured using a least squares fit to an exponential yielding a time constant of $1.138 \mathrm{~ms}$ $(0.088 \% / \mu s)$.

\subsection{Configuration}

The present design is focused on developing a prototype "smart instrument" employing a PC, and mating to it with a special digital interface motherboard designed by LANL and inserted into a PCI slot. This interface will provide all of the logic necessary to move data to the PC memory by DMA transfer, develop event timing, digitizer clock signals, produce time-stamp information, down-load gain and other data required by the analog-front-end that will mount on the digital interface mother board as a daughter board. The interface to the control system will be through the PC.

The analog-front-end electronics includes the signal conditioning, filtering, and digitizers. The signal is amplitude adjusted to provide an appropriate signal to noise ratio for the digitizer. A $7 \mathrm{MHz}$ Gaussian filter provides band limiting with no overshoot and minimal distortion. The amplifier chain provides additional band limiting to $35 \mathrm{MHz}$ to achieve good anti-aliasing characteristics. A. 14 bit $-65 \mathrm{MHz}$ (AD6644) digitizer has been selected to convert the signal to digital data. This is a bipolar converter to accommodate the expected signal droop during the $1 \mathrm{~ms}$ macro-pulse.

\subsection{Signal Conditioning}

To accommodate the large dynamic range expected in the Ring and RTBT, amplifier protection and gain switching require special attention. The MEBT, Linac, 
and HEBT will not require gain changing, and the signal amplification stage is straightforward. A search for high speed, switches and amplifiers capable of handling the large input signal levels for the Ring and RTBT did not prove fruitful. Instead, at present, an attenuator chain and protected amplifier technique is employed. The amplifierinput circuits are diode protected by back-to-back Schottky diodes. To achieve fast gain changes, a switched amplifier is used. Amplifiers were located that could be disabled by a digital control (OPA3860 \& OPA 3861). They switch in less than 100 ns permitting gain changes to be made during the "gap" time. This would permit gain changing without losing a turn. To handle the $60 \mathrm{~dB}$ dynamic range, maintain a signal to noise ratio $>200: 1$ and provide "headroom" for unexpectedly large signals a total of four gains are used.

\subsection{Signal Compensation}

As mentioned earlier, the transformer droop has been compromised to accommodate a short rise time. To properly compute average current and charge for the $1 \mathrm{~ms}$ macro-pulse, the droop and baseline must be restored.

A digital compensation technique has proven to work well and is described here. The transformer transfer function has two dominant poles, a low frequency cut-off and a high frequency cut-off. Multiplication by a transfer function with a zero and a pole can compensate for the undesired transformer low-frequency pole. Setting the compensation filter zero equal to the transformer pole will cancel it. Replacing it with a new pole will set the desired new low frequency response. This is accomplished by carefully measuring the transformer droop time constant. This number is then used to create a zero at this frequency in a compensation filter. The desired low frequency time constant of 1 second $(0.1 \% / \mathrm{ms})$ is used for the compensation filter pole.

The compensation filter is implemented as an IIR filter using a bilinear transformation [4].

$$
\begin{aligned}
& \mathrm{Y}(\mathrm{n})=\left\{1 /\left(2 / \mathrm{T}+1 / \tau_{2}\right)\right\}\left\{\mathrm{y}(\mathrm{n}-1)\left(2 / \mathrm{T}-1 / \tau_{2}\right)+\mathrm{x}(\mathrm{n})\left(2 / \mathrm{T}+1 / \tau_{1}\right)\right. \\
& \left.+\mathrm{x}(\mathrm{n}-1)\left(-2 / \mathrm{T}+1 / \tau_{1}\right)\right\}
\end{aligned}
$$

Where; $\mathrm{T}$ is the sampling time interval, $1 / \tau_{1}$ is the transformer lower cut-off radian frequency, and $1 / \tau$, is the desired new transformer lower cut-off radian frequency

Figure 1 shows raw data taken from a prototype $\Gamma \mathrm{CT}$ stimulated by a gated burst of pulses $645 \mathrm{~ns}$ wide with a $945 \mathrm{~ns}$ period. The droop is quite evident showing the signal drooping to near $37 \%$ of its initial value in $1 \mathrm{~ms}$. Two different compensation zeros are shown to demonstrate the effect of over compensation by about $14 \%$ ( $\tau=1 \mathrm{~ms}$ ) vs correct compensation $(\tau=1.138 \mathrm{~ms})$. This shows an effective lower cut-off frequency near $0.2 \mathrm{~Hz}$. The compensated response still has a droop $(0.1 \% / \mathrm{ms})$. The baseline must be restored to allow proper integration of the current to calculate charge. The baseline restoration process now is simplified since a sample of the
DC level just prior to the compensated macro-pulse is representative of the baseline. This level can be subtracted from the compensated macro-pulse, restoring the baseline to zero.

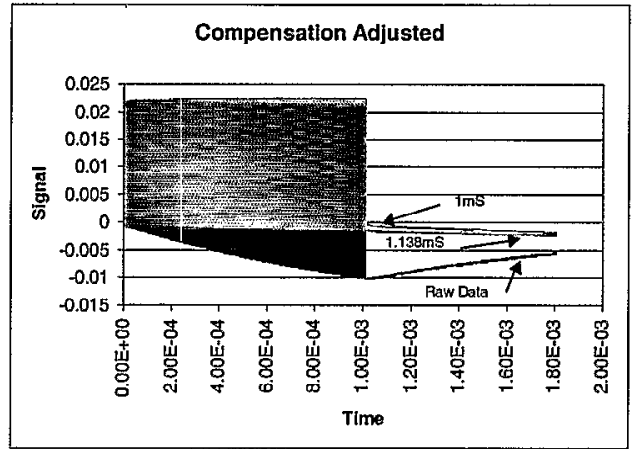

Figure 1: Droop compensated signal.

\subsection{Transformer droop constant calculation}

To properly compensate the transformer, the droop time constant must be accurately measured. Any changes due to temperature or other influences should be properly accounted for. The key to calculating the droop time constant is to obtain good statistical data to minimize the effect of noise in the answer. This can be accomplished by using the raw transformer signal "tail" available at the end of each macro-pulse, or by injecting a test pulse into the transformer calibration winding. This "tail", a portion visible in figure 1 , shows the characteristic droop recovery exponential decay. Data taken during this "tail" is processed by a least square fitting routine to fit it to an exponential decay. There is a total of about $15.66 \mathrm{~ms}$ available to make this calculation before the next macropulse occurs. By using many data points the effect of noise is reduced. The result is a good estimate of the droop time constant for the individual transformer being compensated. Any changes will be updated periodically, and new constants loaded into the IIR compensation filter.

\subsection{Commonality}

To provide commonality the transformer type has been established as the Bergoz ${ }^{\circledR}$ FCT discussed earlier. The goal is to develop an analog front end that is sufficiently flexible to accommodate the single gain requirements of the MEBT, Linac, and HEBT, yet include gain changing for the Ring and RTBT. To adequately sample data to investigate the chopper edges (about 10ns rise time), a high speed digitizer is required. The Ring signal will have a slower rise time after a number of turns, and is conditioned by a 5 pole $7 \mathrm{MHz}$ Gaussian filter. A digitizer has been selected to provide an integral accuracy better than $0.1 \%$, requiring a sampling rate greater than $32 \mathrm{MSa} / \mathrm{S}$. Included in the electronics will be a provision for a wide-band output to investigate the chopper edge (> $100 \mathrm{MHz}$ bandwidth is required). To accomplish this a wide-band amplifier, and a simple resistive splitter are presently under investigation. 
A digital interface is under development at LANL to be used with the Linac BPM electronics. This digital interface is also expected to be used for the BCM system, and the Ring BPM electronics. To provide sufficient digital interface flexibility to adapt this interface for all of the potential uses an industry pack (IP) is included in its design. This industry pack will provide timing information, digitizing clocks, and gain control capabilities for the BCM system. A listing of gains is shown in Table 1.

Table 1: Gain

\begin{tabular}{|c|c|c|}
\hline Turn & Gain & Resolution \\
\hline 1 (up to Ring) & 20 & $2500: 1$ \\
\hline $1-6$ & 3.33 & $416: 1$ \\
\hline $7-84$ & 0.24 & $208: 1$ \\
\hline $85-1060$ & 0.019 & $200: 1$ \\
\hline
\end{tabular}

\section{SUMMARY}

The SNS beam current monitor system is still under development. Resolution requirements have been relaxed to allow three gains to cover the $60 \mathrm{~dB}$ dynamic range in signals expected in the Ring and RTBT. A higher gain will be provided for the single gain requirement of the MEBT, Linac, and HEBT lines. In an effort to provide commonality and utilize the digital interface being developed by LANL, the interface has been made more flexible to accommodate timing and gain setting requirements of the BCM system. A digital droop compensation algorithm has been tested and the results presented. At the time of this writing prototype hardware is under construction.

\section{REFERENCES}

[1] M. Kesselman, et-al, "SNS Project-Wide Beam Current Monitors", BIW 2000, Cambridge MA, May 811,2000

[2] M. Kesselman, et-al, "SNS Project-Wide Beam Current Monitors", EPAC 2000, Vienna, Austria, June 26-30, 2000

[3]http://www.bergoz.com/

[4] C. Britton Rorabaugh "Digital Filter Designer's Handbook" McGraw-Hill, 1993, Ch15. 